\title{
Application of systems modeling and risk assessment to address real-world decision-making challenges
}

\author{
Z. A. Collier ${ }^{1} \cdot$ J. H. Lambert ${ }^{1} \cdot$ I. Linkov ${ }^{2}$
}

Published online: 28 October 2015

(C) Springer Science+Business Media New York 2015

The Editorial Board is pleased to present the latest issue of Environment Systems and Decisions. This issue marks the journal's third full year in press. This issue highlights research that crosscuts subject-matter domains, suggesting the diverse applications of decision analysis, risk analysis, and systems modeling.

The issue begins with a literature review on the topic of sustainability and specifically the relative frequency in which specific sustainability frameworks are implemented (Markolf et al. 2015). In an application of decision analysis, Pagsuyoin et al. (2015) describe a case study of a multiattribute decision model for prioritizing point-of-use water treatment technologies for a community in Philippines. Shittu et al. (2015) develop an economic model that characterizes how competitive pressures and environmental regulations influence the investments of firms in energy technologies. Campbell-Arvai and Arvai (2015) describe a set of experiments that investigate how various asymmetric interventions can affect individual-level decision making and result in behavior that has positive environmental benefits. In an effort to make a business case for the recycling of biodegradable municipal solid waste, Fehr and Arantes (2015) present an analysis of the costs and benefits of recycling wastes from restaurants, shopping centers, and merchants in a Brazilian city. Nguyen et al. (2015) use expert interviews and causal mapping to illustrate how

\footnotetext{
Z. A. Collier

zac4nf@virginia.edu

Igor.Linkov@usace.army.mil

University of Virginia, Charlottesville, VA, USA

2 US Army Engineer Research \& Development Center, Concord, MA, USA
}

$凶$ I. Linkov small and medium enterprises in Vietnam can enhance their adaptive capacity to changing environmental conditions. Kepner et al. (2015) build a percolation-based model for determining the optimal number of rules to create an organizational decision-making climate that minimizes the risks posed by insider threats. In the domain of environmental management, Foran et al. (2015) develop a model to assess the potential cumulative effects of anthropogenic activities based on vulnerability of aquatic resources based on water quality, water quantity, and habitat value metrics. Finally, Jovanovic et al. (2015) present a dynamic systems model in an attempt to forecast the market adoption of aviation biofuels.

Collectively, the papers describe how integrated environmental, economic, and social analyses are applicable to pressing social and technical problems. For example, several papers utilize systems-modeling techniques to gain insights into how environmental pressures and economic forces can be optimized in harmony. Other papers focus on how decision making can be used to make more sustainability-focused choices. This integrated, systems-oriented focus is characteristic of the Springer journal Environment Systems \& Decisions. Readers can look forward to ESD special issues on "Decision Analytics in Economic and Financial Systems" and "Risk Around the World" in the coming year.

\section{References}

Campbell-Arvai V, Arvai J (2015) The promise of asymmetric interventions for addressing risks to environmental systems. Environ Syst Decis. doi:10.1007/s10669-015-9566-1

Fehr M, Arantes CA (2015) Making a case for recycling biodegradable municipal waste. Environ Syst Decis. doi:10.1007/s10669015-9568-z 
Foran CM, Narcisi MJ, Bourne AC, Linkov I (2015) Assessing cumulative effects of multiple activities in New England Watersheds. Environ Syst Decis 35(4). doi:10.1007/s10669015-9575-0

Jovanovic A, Klimek P, Quintero FA (2015) Forecast for the use of alternative fuels in aviation under environmental constraints and volatile market conditions. Environ Syst Decis 35(4). doi:10. 1007/s10669-015-9573-2

Kepner J, Gadepally V, Michaleas P (2015) Percolation model of insider threats to assess the optimum number of rules. Environ Syst Decis. doi:10.1007/s10669-015-9571-4

Markolf SA, Klima K, Wong T (2015) Adaptation frameworks used by United States decision-makers: a literature review. Environ Syst Decis. doi:10.1007/s10669-015-9572-3
Nguyen NH, Beeton RJS, Halog A (2015) A systems thinking approach for enhancing adaptive capacity in small- and mediumsized enterprises: causal mapping of factors influencing environmental adaptation in Vietnam's textile and garment industry. Environ Syst Decis. doi:10.1007/s10669-015-9570-5

Pagsuyoin SA, Santos JR, Latayan JS, Barajas JR (2015) A multiattribute decision-making approach to the selection of point-ofuse water treatment. Environ Syst Decis. doi:10.1007/s10669015-9567-0

Shittu E, Parker G, Jiang X (2015) Energy technology investments in competitive and regulatory environments. Environ Syst Decis. doi:10.1007/s10669-015-9569-y 